\title{
Corela
}

Cognition, représentation, langage

HS-3 | 2006

Les verbes d'apparence

\section{« Expression de l'apparence » : essai collectif de synthèse}

\section{Alain Delplanque}

\section{(2) OpenEdition}

Journals

\section{Édition électronique}

URL : http://journals.openedition.org/corela/1260

DOI : $10.4000 /$ corela. 1260

ISSN : 1638-573X

\section{Éditeur}

Cercle linguistique du Centre et de l'Ouest - CerLICO

\section{Référence électronique}

Alain Delplanque, « «Expression de l'apparence » : essai collectif de synthèse », Corela [En ligne], HS-3 | 2006, mis en ligne le 13 mars 2006, consulté le 19 avril 2019. URL : http:// journals.openedition.org/corela/1260; DOI : 10.4000/corela.1260

Ce document a été généré automatiquement le 19 avril 2019

\section{(c) (i) (2)(2)}

Corela - cognition, représentation, langage est mis à disposition selon les termes de la licence Creative Commons Attribution - Pas d'Utilisation Commerciale - Partage dans les Mêmes Conditions 4.0 International. 


\title{
«Expression de l'apparence »: essai collectif de synthèse
}

\author{
Alain Delplanque
}

1 Dès le début de nos travaux, A. Cambourian a souligné le rapport problématique que l'expression de l'apparence entretient avec l'attribution simple «La pièce est vide / la pièce semble vide, etc ». D'un côté, l'apparence exprime bien un jugement qualitatif, marqué, dans les quatre langues étudiées, par des verbes d'état ou de format compact et pouvant être suivis d'un adjectif. De l'autre côté, l'apparence se distingue de la prédication simple par son caractère indirect ou distancié. Cette distanciation est cependant exempte de l'effet dubitatif qui caractérise notamment certains verbes modaux : «Pierre semble malade » ne veut pas dire la même chose que «Pierre doit être malade ».

2 Sur proposition de S. Osu (communication personnelle), nous avons caractérisé cette distanciation comme une prédication "décalée ». Ce qui a soulevé la question de savoir « entre quoi et quoi » ce décalage intervient. Il y a certes un écart entre l'apparence et la réalité ou plutôt entre l'extérieur de la réalité et son intérieur : une telle référence à la topologie est d'ailleurs suggérée par la morphologie de « aus-sehen » en allemand et celle de "vy-gliadet' » en russe. Mais ce décalage dépend en même temps de la position de l'observateur. En fait, l'apparence fait intervenir trois pôles que C. Agafonov appelle : « réalité, représentation et énonciation ». À partir de là, on peut distinguer deux types de verbes d'apparence, selon que le décalage intervient entre tel et tel pôle de ce tryptique.

3 Le pôle « réalité » appelle cependant quelques explications, dans la mesure où linguistes et philosophes ont tendance à considérer le réel comme extérieur au langage et à la pensée et, partant, sans pertinence dans la réflexion théorique. À cela, il est répondu que l'énonciation travaille à plusieurs niveaux. Certes, tout ce qui énonçable est déterminé par rapport aux coordonnées de celui qui parle (origine Sito, So, To), mais on ne peut pas faire comme si l'énonciateur ne parlait de rien: nous parlons bel et bien de "procès " impliquant des objets et des événements. Ce procès n'est pas la réalité, mais un «filtrage » linguistique de la réalité (cf. Fuchs \& Léonard 1979), une construction de 
l'esprit qui s'effectue au niveau Sit2 (S2, T2). Dans cette perspective, le décalage caractéristique de l'apparence est un cas particulier de filtrage de la réalité

Les langues connues, disions-nous, connaissent deux manières de formuler une impression sur le réel : le « jugement » pur dont l'énonciateur S est la source explicite, et «l'apparence » dont la source est l'objet du monde réel X. Dans une langue africaine comme le dagara, on a les deux solutions suivantes :

5 - soit on dit (0)

\begin{tabular}{|l|l|l|l|l|l|}
\hline n & manina & ke & X & kpéména & / bala \\
\hline je & mesure & que & X & être fort & / être fatigué \\
\hline
\end{tabular}

J'estime, je trouve que X est beau / fatigué

$6 \quad$ - soit on dit (1)

\begin{tabular}{|l|l|l|l|l|l|}
\hline $\mathrm{X}$ & gmena & men & $\mathrm{u}$ & kpéména & / bala \\
\hline $\mathrm{X}$ & frappe & comme & il & être fort & / être fatigué \\
\hline
\end{tabular}

«La mine de X est frappante comme s'il est gros / fatigué »

$\mathrm{X}$ a l'air gros / fatigué

7 Dans ce système minimal, l'énonciateur $S$ ne peut s'exprimer que s'il est sujet comme en (0) ; dans le cas contraire (1), le sujet est $\mathrm{X}$ et l'énonciateur est nécessairement implicite. Seul (1) exprime une apparence, phénomène qui se caractérise donc par une certaine mise à distance de l'énonciateur. Celle-ci peut occasionner une évacuation pure et simple de l'énonciateur (comme en dagara) ou son transfert en position de destinataire (comme en français : " X m'a l'air petit, fatigué »). Ceci justifie la distinction établie par G. Col entre l'observateur «constructeur» et l'observateur «récepteur» avec les visées correspondantes :

(2 a) perception

$$
\text { (S) } \rightarrow X
$$

look, wgliadet', aus-sehen
(2 b) effet

$$
x \rightarrow(S)
$$

faire, wirken

8 La présence de $\mathrm{S}$ indique que le procès est explicitement repéré par rapport à l'énonciateur, ce qui permet d'effectuer divers calculs à partir des coordonnées spatiotemporelles de l'énonciation. Ceci explique d'abord la compatibilité des apparences de type 1 avec l'aspectualité propre aux attributs verbaux ( $\mathrm{X}$ a l'air de souffrir / d'avoir souffert »). Ensuite, cette temporalité engendre des sous-classes de verbes impliquant une chronologie. Notamment, dans les quatre langues, l'un des verbes est un verbe de processus qui traduit une "impression seconde" que l'énonciateur exprime au terme d'un examen attentif ou d'une période de doute. Il s'agit de : « appear, apparaître, erscheinen et de predstavliat'sia ». 
9 À partir du couple primitif jugement / apparence, on peut calculer deux types de prédication hybride, que A. Delplanque appelle «jugement d'après les apparences », et qu'on obtient par diverses opérations de diathèse impliquant l'évacuation de l'énonciateur S :

\section{(2 a) perception}

$$
\text { (S) } \rightarrow x
$$

look, wgliadet', aus-sehen
(2 b) effet

$$
x \rightarrow(S)
$$

faire, wirken perception visuelle pris au sens figuré (type $2 \mathrm{a}: \mathrm{I}$ look at the picture / the picture looks nice). Dans ces trois langues (allemand, anglais, russe), ce verbe n'est jamais suivi d'une complétive et ne permet d'appréhender le réel que du point de vue qualitatif ; il a de plus des affinités avec la comparaison (look like, look as if). L'anglais permet de marquer l'énonciateur transposé en destinataire (« it looks nice to me; John looks the right guy to me »). Mais en russe et en allemand, ce verbe ne peut être suivi d'un datif (*mne, ${ }^{*}$ mir), ce qui suggère que la modulation est ici liée, non pas tant à un débat inter-sujets, mais davantage au caractère partiel ou imparfait de la vision humaine.

11 Par ailleurs, en français et en allemand, l'un des verbes d'apparence est un verbe d'action détourné (type $2 \mathrm{~b}$ : faire, wirken) qui traduit, en quelque sorte, l'effet produit sur un énonciateur "passif" par le sujet dans son environnement. Ces verbes permettent une qualification d'une subjectivité telle qu'elle met le débat hors de propos: «ça fait paysan ».

12 Ces différentes visées ou, pour reprendre l'expression de G. Col, ces divers modes d'émergence du sens dépendent de la mise en scène verbale, ou de la manière dont «l'espace mental» est structuré. De ce fait, le décalage qui caractérise l'apparence connaît plusieurs degrés. Les formules de type (2) impliquent une certaine "proximité " entre l'énonciateur et le réel. Ce terme ne doit pas être pris au sens métrique, mais au sens topologique: $\mathrm{S}$ et $\mathrm{X}$ ne sont pas séparés, $\mathrm{X}$ est dans le même espace que $\mathrm{S}$. Corollairement, l'apparence caractérise la surface visible des choses (la frontière entre l'Intérieur et l'Extérieur). C'est alors que l'énonciateur (S) s'efface au profit de l'objet X, ce qui ne signifie nullement une absence de subjectivité.

13 En revanche, dans le type (1), la position de l'énonciateur S est cette fois en « rupture » avec la réalité $\mathrm{X}$. Ce terme est également à prendre au sens topologique : l'apparence, en quelque sorte, faisant écran entre la réalité et l'énonciation. Ce "solipsisme " qui caractérise le rapport de l'objet au sujet-pensant pourrait expliquer la réflexivité qui apparait en russe avec tous les verbes du type «kazat'sia » («X se-montre à moi, il sepense à moi que XP »). Cette "distanciation" pourrait aussi expliquer le caractère dialectique de l'assertion avec ce type de verbe: les apparences sont trompeuses ( $c f$. «mereshchit'sja » et "chudit'sja » en russe), ou voici ce que j'en dis pour l'instant / après-coup ( $c f$. contraste entre "paraitre / apparaître » en français, entre "scheinen / erscheinen » en allemand, entre "kazat'sia » et "predstavljat'sia » en russe). Avec une nuance, cependant, car en dehors de «avoir l'air », les verbes du type 1 («sembler, seem, kazat'sia, scheinen ») admettent deux tournures paraphrastiques : 
(1a) sujet personnel + infinitif

$$
x \rightarrow S
$$

Pierre me semble dormir (1b) sujet impersonnel + complétive

$$
() \rightarrow S
$$

II me semble que Pierre dort. L'impersonnel étant interprété comme un sujet vide, on réalise alors un écart maximal entre le réel X et l'énonciateur S. Plus exactement, la construction $1 \mathrm{~b}$ a pour effet de désynchroniser l'apparence et sa validation : le procès est préconstruit mais sa prise en charge reste en attente. compact, ils n'ont pas les mêmes valences syntaxiques et engendrent des énoncés de formats divers selon le type de l'attribut qui les suit (N, A ou V). En français, on a vu la complémentarité de "faire, ressembler, avoir l'air » selon que l'attribut est compact, dense ou discret. À cet égard, on a également vu le rôle joué par la connexion de l'attribut dans les quatre langues (like, wie, de, à, etc.).

Le russe est à ce propos particulièrement délicat, avec le contraste entre l'instrumental et le comparatif «kak ». L'instrumental (cas " périphérique ») permet d'établir une distance entre le sujet et son attribut : on pourrait parler d'une discrétisation, compatible avec les deux types d'apparence. Que l'attribut soit un adjectif ou un nom, l'instrumental marque alors une subjectivité plus grande (jugement de valeur). En revanche, "kak» n'est possible qu'avec un attribut nominal et seulement dans le cas de "vygliadet'», car la comparaison consiste à rapprocher deux termes au départ séparés, ce qui a pour effet de densifier le domaine, opération incompatible avec les propriétés discrètes des apparences du type 1. Corollairement, "kak» marque une subjectivité moins grande et évoque davantage les propriétés du réel. D'où la paire «elle a l'air d'une princesse / elle ressemble à la princesse Diana ", qu'on trouve aussi bien en russe qu'en français. On a ainsi deux mouvements inverses, grosso modo :

- avec « Marie a l'air d'une princesse », je dis que Marie est une princesse, mais j'ajoute qu'elle n'en a que l'apparence.

- avec « Marie ressemble à la Princesse », je dis que Marie n'est pas la Princesse, mais j'ajoute qu'elle n'en est pas loin.

De ce qui précède, on peut conclure que l'apparence est exprimée de manières fort diverses à l'intérieur d'une même langue, et à plus forte raison d'une langue à l'autre. Le plus souvent, tel verbe n'a pas de correspondant exact dans une autre langue ( $c f$. le rapport entre l'anglais « look » et les verbes français « avoir l'air, faire, ressembler »). Et surtout, toutes ces langues ne distinguent pas les mêmes nuances et n'activent pas les mêmes (sous-) catégories.

Toutefois, les formules ci-dessus tracées suggèrent une logique générale qui permet de dériver un ensemble de cas de figure à partir d'une primitive (le jugement de type 0). De cet ensemble, telle langue peut retenir plus ou moins de structures que telle autre langue. À cet égard, l'allemand a ceci d'original qu'il possède les deux formules $2 a$ et $2 b$. C'est sans doute ce qui justifie l'optimisme d'A. Cambourian quand il parle d'un « système clos »... 